\title{
A biomarker study in long-lasting amnestic mild cognitive impairment
}

\author{
Chiara Cerami ${ }^{1,2^{*}}$, Alessandra Dodich ${ }^{1,2}$, Sandro lannaccone ${ }^{2}$, Giuseppe Magnani ${ }^{3}$, Roberto Santangelo ${ }^{3}$, \\ Luca Presotto ${ }^{4}$, Alessandra Marcone ${ }^{2}$, Luigi Gianolli ${ }^{4}$, Stefano F. Cappa ${ }^{5,6}$ and Daniela Perani ${ }^{1,4,7}$
}

\begin{abstract}
Background: Mild cognitive impairment (MCl) is a heterogeneous syndrome resulting from Alzheimer's disease (AD) as well as to non-AD and non-neurodegenerative conditions. A subset of patients with amnestic $\mathrm{MCl}$ (aMCl) present with an unusually long-lasting course, a slow rate of clinical neuropsychological progression, and evidence of focal involvement of medial temporal lobe structures. In the present study, we explored positron emission tomography (PET) and cerebrospinal fluid (CSF) biomarkers in a sample of subjects with aMCI with such clinical features in order to provide in vivo evidence to improve disease characterisation in this subgroup.

Methods: Thirty consecutive subjects with aMCl who had long-lasting memory impairment (more than 4 years from symptom onset) and a very slow rate of cognitive progression were included. All subjects underwent fluorodeoxyglucose-positron emission tomography (FDG-PET) metabolic imaging. A measure of cerebral amyloid load, by PET and/or CSF, was obtained in 26 of 30 subjects. The mean clinical follow-up was $58.3 \pm$ 10.1 months.

Results: No patient progressed to dementia during the follow-up. The typical AD FDG-PET pattern of temporoparietal hypometabolism was not present in any of the subjects. In contrast, a selective medial temporal lobe hypometabolism was present in all subjects, with an extension to frontolimbic regions in some subjects. PET imaging showed absent or low amyloid load in the majority of samples. The values were well below those reported in prodromal AD, and they were slightly elevated in only two subjects, consistent with the CSF $\beta$-amyloid (1-42) protein values. Notably, no amyloid load was present in the hippocampal structures.

Conclusions: FDG-PET and amyloid-PET together with CSF findings questioned AD pathology as a unique neuropathological substrate in this a $\mathrm{MCl}$ subgroup with long-lasting disease course. The possibility of alternative pathological conditions, such as argyrophilic grain disease, primary age-related tauopathy or age-related TDP-43 proteinopathy, known to spread throughout the medial temporal lobe and limbic system structures should be considered in these patients with $\mathrm{MCl}$.
\end{abstract}

Keywords: Positron emission tomography, Mild cognitive impairment, Alzheimer's disease, Medial temporal lobe dysfunction, FDG-PET, Amyloid-PET

\footnotetext{
* Correspondence: cerami.chiara@hsr.it

${ }^{1}$ Division of Neuroscience, San Raffaele Scientific Institute, Milan, Italy

${ }^{2}$ Clinical Neuroscience Department, San Raffaele Turro Hospital, Milan, Italy

Full list of author information is available at the end of the article
}

(c) The Author(s). 2018 Open Access This article is distributed under the terms of the Creative Commons Attribution 4.0 International License (http://creativecommons.org/licenses/by/4.0/), which permits unrestricted use, distribution, and reproduction in any medium, provided you give appropriate credit to the original author(s) and the source, provide a link to the Creative Commons license, and indicate if changes were made. The Creative Commons Public Domain Dedication waiver (http://creativecommons.org/publicdomain/zero/1.0/) applies to the data made available in this article, unless otherwise stated. 


\section{Background}

Mild cognitive impairment (MCI) is a heterogeneous syndrome that can be due to Alzheimer's disease (AD) and non-AD pathologies [1]. The presence of an early and significant objective deficit of episodic memory is considered the main criterion supporting the diagnosis of typical $\mathrm{AD}$ and the best cognitive predictor of the development of $\mathrm{AD}$ dementia $[2,3]$. Though the amnestic syndrome of hippocampal type is the most typical presentation in prodromal $\mathrm{AD}$, impairments in delayed recall tasks may be present in individuals with non-AD disorders, such as the behavioural variant of frontotemporal dementia (bvFTD) [4], argyrophilic grain disease (AGD) $[5,6]$ and the recently identified suspected non-AD pathology (SNAP) [7-9].

Subjects with selective long-term memory impairment and a relatively stable or very slowly progressing (up to decades) condition have been reported [10, 11]. This condition has been considered up to now as a phenotypical expression of a focal medial temporal lobe dysfunction possibly due to $\mathrm{AD}$ pathology [10-12]. In particular, patients with $\mathrm{AD}$ with a limbic predominant deposition of tau protein present with a specific phenotype (e.g., old age at onset, predominant amnestic syndrome) and selective medial temporal lobe atrophy visualised by magnetic resonance imaging (MRI) compared with the other AD variants (i.e., typical AD and hippocampus-sparing AD) [13].

Recently, patients with MCI with such a clinical phenotype and no clear in vivo evidence of AD pathology were reported in the literature as part of the heterogeneous clinical group labelled SNAP [8, 9, 14-17]. This is a biomarker-based concept applied to individuals with evidence of neurodegeneration in the absence of cerebral amyloid load [9]. This definition reflects the notion that pathologies other than AD may underlie neurodegenerative changes, as revealed by cerebrospinal fluid (CSF) and fluorodeoxyglucose-positron emission tomography (FDG-PET) biomarkers, in subgroups of patients clinically presenting with an AD-like phenotype.

In the present study, we assessed FDG-PET brain hypometabolism and cerebral amyloid load by PET imaging as well as CSF $\beta$-amyloid $(1-42)$ protein $\left(A \beta_{42}\right)$ and tau values in a sample of subjects with MCI with predominant episodic memory impairment and a very slow rate of progression. The aim was to provide in vivo evidence to improve disease characterisation in this MCI subgroup.

\section{Methods}

\section{Participants}

The sample included 30 subjects (mean age $74.1 \pm 4$. 8 years; mean education level $10.3 \pm 4.5$ years; mean disease duration at first evaluation $44.5 \pm 25.5$ months) fulfilling the Petersen criteria [1] for amnestic MCI (aMCI) and characterised by (1) a predominant episodic memory impairment, (2) a long-term clinical course (i.e., more than 4 years) and (3) a slow rate of progression of memory deficits. All subjects were consecutively referred to the neurology centres of San Raffaele Hospital (Milan, Italy). All of the included patients had a 3 to 5 years of clinical follow-up (i.e., $58.3 \pm 10.1$ months).

Conventional MRI was used to exclude the presence of white matter hyperintensities and lacunes of cerebrovascular origin as causes of cognitive impairments. Upon conventional inspection of MRI scans, hippocampal atrophy was found in the majority of subjects, without a clear radiological picture of hippocampal sclerosis (i.e., reduction of hippocampal volume with abnormal shape of mesial temporal lobe structures observed on T1-weighted images and increased signal intensity on T2-weighted and fluid-attenuated inversion recovery images) [18]. All of the included subjects had an FDG-PET scan, and 26 of 30 subjects with MCI had an amyloid biomarker evaluation (i.e., either with amyloid-PET and/or CSF A $\beta_{42}$ measure). See Tables 1 and 2 for details.

\section{CSF measures}

CSF was obtained from 20 of 30 subjects near the time of first clinical evaluation ( $<3$ months) by lumbar puncture in the L3-L4 or L4-L5 interspace. The procedure was performed early in the morning. No serious adverse events were reported. CSF $(8-10 \mathrm{ml})$ was collected in sterile polypropylene tubes. Part of it was used to determine routine chemical parameters (leucocyte and erythrocyte cell count, glucose measurement, protein total content). The remaining CSF was centrifuged for $10 \mathrm{~min}$ at $4000 \times g$ at $4{ }^{\circ} \mathrm{C}$ and stored at $-80{ }^{\circ} \mathrm{C}$ until analysis to ensure the stability of the CSF biomarkers. Measurement of CSF $A \beta_{42}$, total tau (t-tau) and phosphorylated tau (p-tau) levels was performed using commercial available enzyme-linked immunosorbent assay (ELISA) kits according to the manufacturer's protocol and blinded to clinical data. Normal values were set $\geq 500 \mathrm{ng} / \mathrm{L}$ for $\mathrm{A} \beta_{42}$ values, $\leq 450 \mathrm{ng} / \mathrm{L}$ (if age was $51-70$ years) or $<500 \mathrm{ng} / \mathrm{L}$ (if age was $>71$ years) for t-tau values and $\leq 61 \mathrm{ng} / \mathrm{L}$ for $\mathrm{p}$-tau values, according to the ELISA kit guidelines and literature recommendations [19].

\section{FDG-PET imaging}

FDG-PET scans were acquired in 30 of 30 patients near the time of first clinical evaluation ( $<3$ months) according to European Association of Nuclear Medicine guidelines [20]. FDG-PET was performed in each subject at the Nuclear Medicine Unit, San Raffaele Hospital (Milan, Italy), with the Discovery STE multi-ring PET-computed tomography (CT) system (GE Medical Systems, Milwaukee, WI, USA). Before radiopharmaceutical injection of FDG 
Table 1 Demographic and clinical features of the sample

\begin{tabular}{|c|c|}
\hline & $\begin{array}{l}\text { Patient sample } \\
(n=30)\end{array}$ \\
\hline Female/male ratio & $12 / 18$ \\
\hline Age in years (mean $\pm S D)$ & $74.1 \pm 4.8$ \\
\hline Age range in years & $65-84$ \\
\hline Years of education (mean \pm SD) & $10.3 \pm 4.5$ \\
\hline $\begin{array}{l}\text { Disease duration in months at first } \\
\text { evaluation (mean } \pm S D \text { ) }\end{array}$ & $44.5 \pm 25.5$ \\
\hline Months of follow-up (mean \pm SD) & $58.3 \pm 10.1$ \\
\hline $\begin{array}{l}\text { Disease duration in months at follow-up } \\
\text { (mean } \pm \text { SD) }\end{array}$ & $102.8 \pm 28.1$ \\
\hline $\begin{array}{l}\text { MMSE adjusted score at first evaluation } \\
\text { (mean } \pm \text { SD) }\end{array}$ & $26.5 \pm 2.1$ \\
\hline $\begin{array}{l}\text { MMSE adjusted score at follow-up } \\
\text { (mean } \pm \text { SD) }\end{array}$ & $25.1 \pm 2.5$ \\
\hline $\begin{array}{l}\text { CDR Sum of Boxes at first evaluation } \\
\text { (mean } \pm \text { SD) }\end{array}$ & $1.91 \pm 0.6$ \\
\hline $\begin{array}{l}\text { CDR Sum of Boxes at follow-up } \\
\text { (mean } \pm \text { SD) }\end{array}$ & $2.9 \pm 1.7$ \\
\hline Diagnosis at first evaluation & 15 s-aMCl, 15 m-aMCl \\
\hline Diagnosis at follow-up & $9 \mathrm{~s}-\mathrm{aMCl}, 21 \mathrm{~m}-\mathrm{aMCl}$ \\
\hline Lumbar puncture (no. of subjects) & $20 / 30$ \\
\hline CSF $A \beta_{42}$ and t-tau/p-tau normal values & $7 / 20$ \\
\hline CSF $A \beta_{42}$ low values & $10 / 20$ \\
\hline CSF p-Tau/t-tau high values & $3 / 20$ \\
\hline Amyloid-PET (no. of subjects) & $16 / 30$ \\
\hline SUVr values from 1.0 to 1.45 & $8 / 16$ \\
\hline SUVr values from 1.45 to 1.80 & $6 / 16$ \\
\hline SUVr values from 1.80 to 1.90 & $2 / 16$ \\
\hline
\end{tabular}

Abbreviations: MMSE Mini Mental State Examination, CDR Clinical Dementia Rating, CSF Cerebrospinal fluid, $A \beta_{42} \beta$-Amyloid (1-42) protein, $t$-tau Total tau, p-tau Phosphorylated tau, s-aMCl Single-domain amnestic mild cognitive impairment, $m$-aMCI Multiple-domain amnestic mild cognitive impairment, PET Positron emission tomography

(usually $185-250 \mathrm{MBq}$ via a venous cannula), subjects were fasted for at least $6 \mathrm{~h}$, and the measured blood glucose threshold was $<120 \mathrm{mg} / \mathrm{dl}$. All images were acquired with an interval between injection and scan start of $45 \mathrm{~min}$ and scan duration of $15 \mathrm{~min}$. Images were reconstructed using an ordered subset expectation maximisation (OSEM) algorithm. Attenuation correction was based on CT scans. Specific software integrated in the scanner was used for scatter correction. All subjects gave written informed consent after detailed explanation of the FDGPET procedure.

Image pre-processing was performed using statistical parametric mapping (SPM; http://www.fil.ion.ucl.ac.uk/ spm/software) according to an optimised SPM procedure with implementation of a standardised SPM FDG dementia-specific template [21] for spatial normalisation. This is an optimised method validated in MCI and different dementia conditions at the single-subject level and showing high accuracy in estimating specific metabolic patterns [22-24]. In detail, images were smoothed with an 8-mm FWHM gaussian kernel; proportional scaling was used to remove inter-subject global variation in PET intensity; and each FDG-PET scan was then tested for relative "hypometabolism" by means of a twosamples $t$ test implemented in SPM in a comparison with a normal FDG-PET image database $(n=112)$ on a voxel-by-voxel basis, with age as a covariate [22]. The FDG-PET image database included data acquired with different PET scanners. However, this did not affect data analysis with the optimised SPM procedure as evaluated and reported by Presotto et al. [25] with a large sample of FDG-PET images acquired with several PET scanners. The statistical threshold for the analysis at single-subject level was set at $p=0.05$, familywise error (FWE)-corrected for multiple comparisons at the voxel level. Only clusters containing more than 100 voxels (i.e., $800 \mathrm{~mm}^{3}$ ) were deemed to be significant.

In order to compute brain metabolic changes at the group level, we also performed a second-level SPM analysis (one-sample $t$ test) in the whole MCI group. The threshold was set at $p<0.05$, FWE-corrected for multiple comparisons at the voxel level. In order to reveal more subtle effects in brain hypometabolism, a $p<0.001$ uncorrected analysis (FWE-corrected at the cluster level) was also performed.

\section{Amyloid-PET imaging}

Amyloid-PET imaging was performed in 16 of 30 subjects using the $\left[{ }^{18} \mathrm{~F}\right]$ florbetaben NeuraCeq ${ }^{\mathrm{Tm}}$ tracer (Piramal Imaging, Berlin, Germany). All amyloid-PET acquisitions were performed within a mean of 3-12 months (i.e., $5.6 \pm 1.9$ months) from the first clinical evaluation. Patients received a single intravenous bolus injection of $315.6 \pm 16.6 \mathrm{MBq}$ of tracer. PET scans were acquired using a hybrid GE Discovery PET/CT 690 system (interval between injection and scan start 90 min; scan duration $15 \mathrm{~min}$ ) [26]. CT scans were used for attenuation correction of PET data. All image reconstruction was performed using a 3D-OSEM algorithm. Images were scaled by division through the respective median cerebellar grey matter voxel intensity, and image processing resulted in regional cortical grey matter tracer uptake values relative to cerebellar grey matter (i.e., standardised uptake value [SUV]). SUVs were calculated for all regions according to the procedure reported by Villemagne et al. [27]. In particular, we considered ROIs in frontal (i.e., dorsolateral, ventrolateral and orbitofrontal regions), superior and inferior parietal, lateral temporal, lateral occipital, and anterior and posterior cingulate cortices. Each SUV was used to derive the composite SUV ratio (SUVr) referenced to the cerebellar 


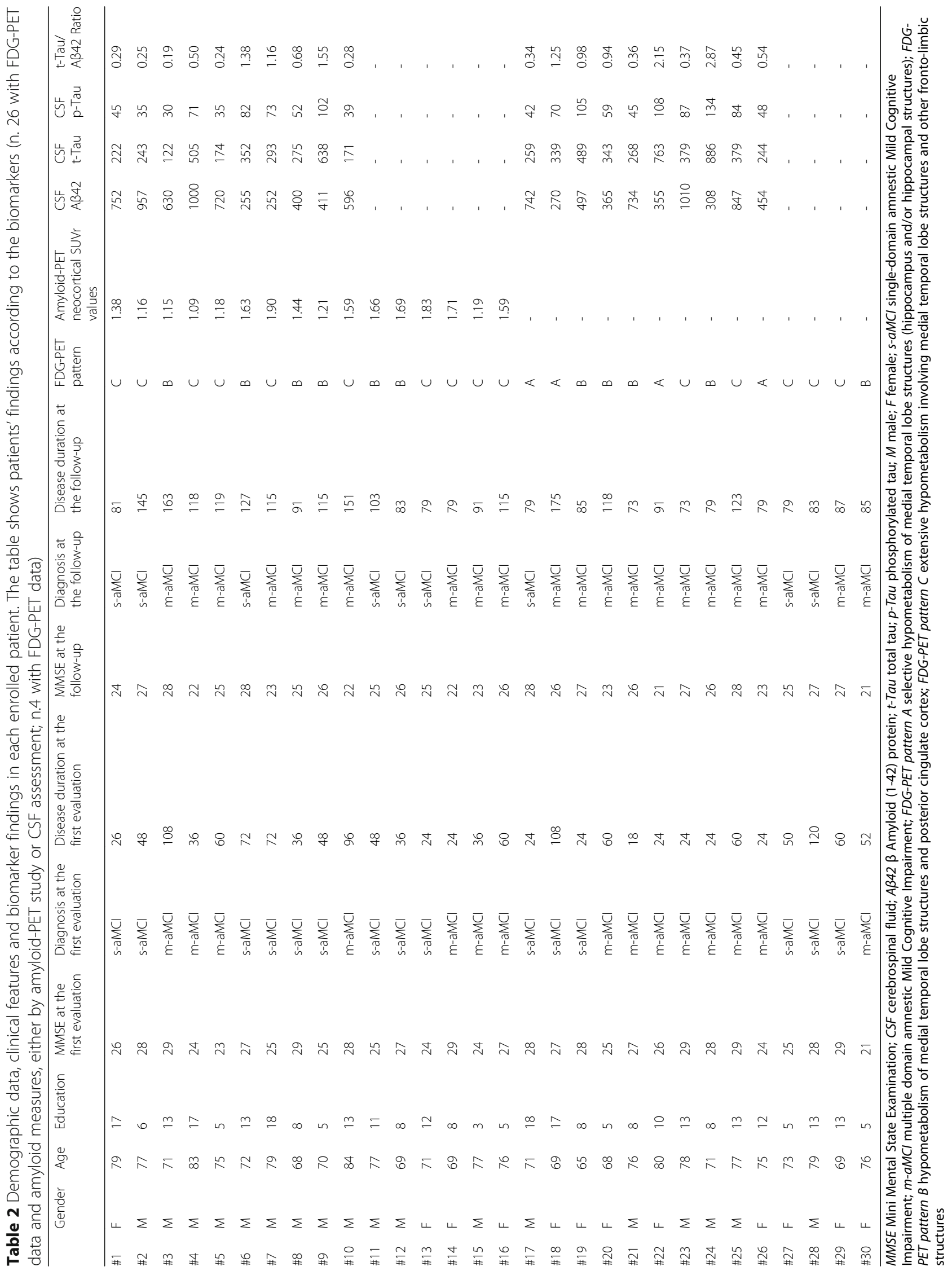


cortex, a region most frequently used in SUV analysis because it is relatively unaffected by $\mathrm{A} \beta$ plaques in sporadic $\mathrm{AD}$ [27]. In addition to the above-mentioned cortical regions suggested in the literature to calculate the composite neocortical SUVr, we selected ROIs in hippocampal structures, amygdala and insula according to the Automated Anatomical Labelling atlas. This further analysis was done in order to test in vivo with PET whether amyloid deposits leading to a major effect on neurodegeneration in these brain regions might selectively exist. The long-lasting disease duration in subjects with MCI with selective longterm memory impairment or relatively stable or very slow progression has indeed been attributed to focal medial temporal lobe dysfunction possibly due to AD pathology [10-12]. Finally, a non-parametric correlation analysis (Spearman's rho) was performed to evaluate the relationship between mean neocortical amyloid-PET SUVr values and CSF $A \beta_{42}$ values in those patients who underwent both amyloid-PET and lumbar puncture.

\section{Results}

\section{Clinical neuropsychological findings}

At the time of the first clinical evaluation, the subjects' age ranged from 65 to 84 years, and both sexes were similarly affected. Fifteen subjects were classified as having singledomain aMCI (s-aMCI), and 15 were classified as having multiple-domain aMCI (m-aMCI). Of note, 21 of 30 subjects showed behavioural disturbances (i.e., anxiety, irritability, aggressiveness, apathy and emotional blunting). At the clinical follow-up (58.3 \pm 10.1 months), none had progressed to dementia. The majority of subjects with aMCI showed stable profiles (i.e., 9 with s-aMCI and 15 with $\mathrm{m}$-aMCI). Mild progression of memory impairment and additional cognitive deficits were found in 6 subjects with s-aMCI, who were reclassified as having $\mathrm{m}$-aMCI. See Tables 1 and 2 for demographic and clinical details.

Subjects with MCI who had some evidence of amyloid deposition $(n=15)$ visualised by CSF or PET imaging (see below) did not show any significant difference in clinical or neuropsychological features compared with amyloid-negative $(n=11)$ subjects, except for the female/male ratio and the disease duration in months, with amyloid-negative subjects showing longer disease duration (Table 3).

\section{CSF findings}

Among the 20 subjects with CSF data available, 7 had completely normal CSF values. Three subjects showed only slight elevation of p-tau levels, suggesting the presence of a neurodegenerative process. The remaining ten subjects showed $A \beta$ values below the cut-off proposed for $A \beta$ positivity [19]. Half of these subjects (i.e., five of ten) had only slightly altered $A \beta$ results, with normal t-tau and p-tau values found in three of them. In these latter three subjects, $\mathrm{MCI}$ due to $\mathrm{AD}$ had a low probability according to the Erlangen Score Algorithm for the interpretation of CSF results $[28,29]$. Pathological $A \beta$ results, with slight alteration of p-tau levels, were present in three subjects.

Table 3 Clinical and neuropsychological features of the $\mathrm{MCl}$ sample grouped according to the evidence (i.e., $\mathrm{A} \beta+\mathrm{MCl}$ ) or the absence (i.e., A $\beta-\mathrm{MCl}$ ) of amyloid pathology by amyloid-PET and/or CSF imaging. Amyloid pathology information was available in $26 / 30 \mathrm{MCl}$ subjects. Values are shown as mean \pm standard deviation

\begin{tabular}{|c|c|c|c|}
\hline & $A \beta+M C l n=15$ & $A \beta-M C l \quad n=11$ & Statistics \\
\hline Female/Male ratio & $8 / 7$ & $11 / 1$ & $p<0.05$ \\
\hline Age in years & $73.5 \pm 5.4$ & $75.3 \pm 4.5$ & $p=0.4$ \\
\hline Years of education & $9.2 \pm 4.8$ & $11.1 \pm 4.9$ & $p=0.3$ \\
\hline Disease duration in months & $30.3 \pm 16$ & $50.3 \pm 29$ & $p<0.05$ \\
\hline \multicolumn{4}{|l|}{ Diagnosis at the first evaluation } \\
\hline Mini Mental State Examination (cut-off = 24) & $25 \pm 3.9$ & $26.5 \pm 2.9$ & $p=0.31$ \\
\hline Token test (cut-off $=26.25$ ) & $30.8 \pm 4.3$ & $30.7 \pm 2$ & $p=0.9$ \\
\hline Phonemic fluency (cut-off $=16$ ) & $24.1 \pm 12.2$ & $20 \pm 7.4$ & $p=0.34$ \\
\hline Semantic fluency (cut-off = 24) & $28.8 \pm 10.5$ & $27.1 \pm 5.7$ & $p=0.64$ \\
\hline Digit Span (cut-off = 3.5) & $5.23 \pm 1.3$ & $5.5 \pm 1.1$ & $p=0.66$ \\
\hline Corsi Span (cut-off = 3.5) & $4.7 \pm 0.8$ & $4.3 \pm 0.7$ & $p=0.25$ \\
\hline Rey Auditory Verbal Learning Test immediate recall (cut-off $=28.5)$ & $19.17 \pm 3.7$ & $20.3 \pm 5.1$ & $p=0.55$ \\
\hline Rey Auditory Verbal Learning Test delayed recall $($ cut-off $=4.68)$ & $1.42 \pm 1.9$ & $1.22 \pm 1.5$ & $p=0.80$ \\
\hline Rey-Osterrieth Complex Figure recall (cut-off $=9.46$ ) & $6.25 \pm 3.1$ & $6.25 \pm 5.2$ & $p=1$ \\
\hline Rey-Osterrieth Complex Figure copy (cut-off $=28.87$ ) & $22.6 \pm 9$ & $28.64 \pm 6.1$ & $p=0.075$ \\
\hline Attentive Matrices (cut-off $=30$ ) & $43.85 \pm 7$ & $45.18 \pm 10.7$ & $p=0.72$ \\
\hline Raven Matrices (cut-off $=17.5$ ) & $24.9 \pm 7.1$ & $22.73 \pm 6.1$ & $p=0.52$ \\
\hline
\end{tabular}


Only two subjects showed clearly pathological results for both $\mathrm{A} \beta$ and $\mathrm{t}$-tau/p-tau, compatible with an AD-like CSF profile [29]. See Table 2 for further details on individualsubject values. The $\mathrm{t}$-tau/A $\beta$ ratio confirmed an $\mathrm{AD}$-like profile in those subjects with clearly pathological $A \beta$ values. Ratios above the cut-off proposed for $\mathrm{AD}$ positivity as reported by Shaw et al. [30] were also found in four of the subjects with an unclear single-biomarker profile. See Table 2 for details on individual-subject values.

\section{FDG-PET imaging findings}

No subject showed the typical AD temporoparietal hypometabolism on the basis of FDG-PET imaging [22-24]. We found instead a consistent pattern of focal hypometabolism involving the hippocampus and/or hippocampal structures in every case. In half of the sample, additional metabolic changes were present in the frontomedial cortex, the amygdala, the posterior part of the insula extending to the parietal operculum and the superior temporal gyrus. Some patients (i.e., 11 of 30) showed posterior cingulate cortex hypometabolism. See Fig. $1 \mathrm{a}$ and $\mathrm{b}$ and Table 2 for details.

The group analysis of FDG-PET data using the SPM procedure showed a bilateral pattern of hypometabolism involving the hippocampal structures spreading to the insula on the left side $(p<0.05$, FWE-corrected for multiple comparisons at the cluster level) (Fig. 1c). At the less stringent statistical threshold of significance (i.e., $p<0.001$ uncorrected, FWE-corrected at the cluster level), we found a more extended hypometabolism only in the medial temporal lobe structures, without any involvement of the lateral temporoparietal regions (Fig. 1d).

\section{Amyloid-PET imaging findings}

Negative or very low levels of tracer uptake were found in the majority of subjects with MCI (Table 2). In particular, 8 of 16 patients had very low composite SUVr values $(1.22 \pm 0.12)$, comparable to those reported in the literature for $\mathrm{A} \beta$-negative healthy control subjects [27, 31-35], and notably well below the proposed cutoff for prodromal AD (i.e., < 1.45) [33]. Low to intermediate SUVr values $(1.45<\mathrm{SUVr}<1.80)$ below the mean values reported in the literature for $A D A \beta$ positivity were found in 6 of 16 subjects (see Fig. 2). Only 2 of 16 subjects had slightly elevated composite SUVr values (i.e., 1.83 and 1.90) (Fig. 2). No regional effect of the tracer retention in the single cortical ROIs included in the composite SUVr measure emerged. Notably, in all subjects with $\mathrm{MCI}$, there was a sparing of the hippocampal structures, amygdala and insula, in which SUVr values were always low overall $(<1.4)$. See Fig. 2 for details.

\section{CSF $A \beta_{42}$ and amyloid-PET imaging correspondence}

Amyloid-PET SUVr and CSF A $\beta_{42}$ values were consistent (Fig. 3). In detail, seven subjects had normal or slightly

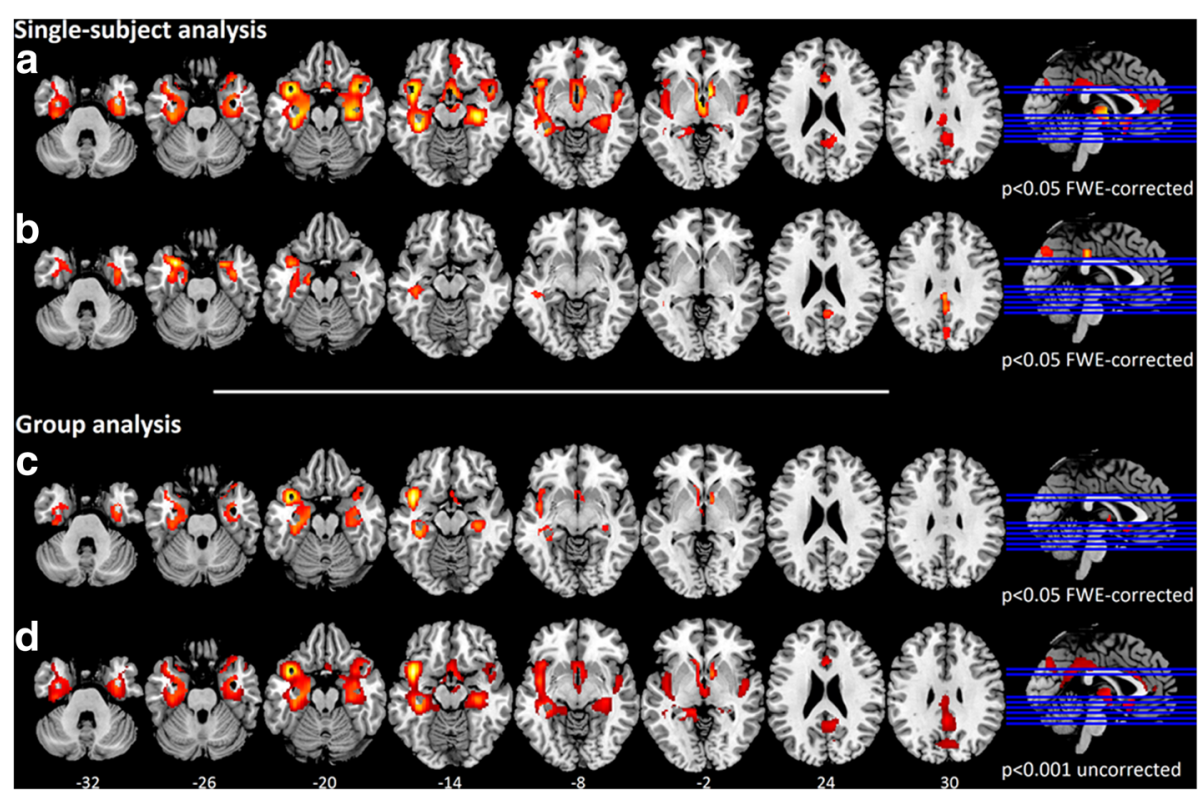

Fig. 1 a and $\mathbf{b}$ Fluorodeoxyglucose-positron emission tomography (FDG-PET) hypometabolic patterns of two example subjects (i.e., statistical parametric mapping [SPM] single-subject analysis: 1 patient vs. 112 control subjects; $p<0.05$, familywise error [FWE]-corrected at the voxel level with $k>100$ voxels) representing (a) extensive hypometabolism in the medial temporal lobe (i.e., hippocampus and hippocampal structures) and limbic structures (amygdala, frontomedial cortex, and insula) and (b) selective medial temporal lobe hypometabolism, more extended on the left side. $\mathbf{c}$ and $\mathbf{d}$ FDG-PET hypometabolic pattern of the whole patient group (i.e., SPM one-sample group analysis; $p<0.05$ FWE-corrected at the voxel level and $p<0.001$ uncorrected at the voxel level, FWE-corrected at cluster level) 


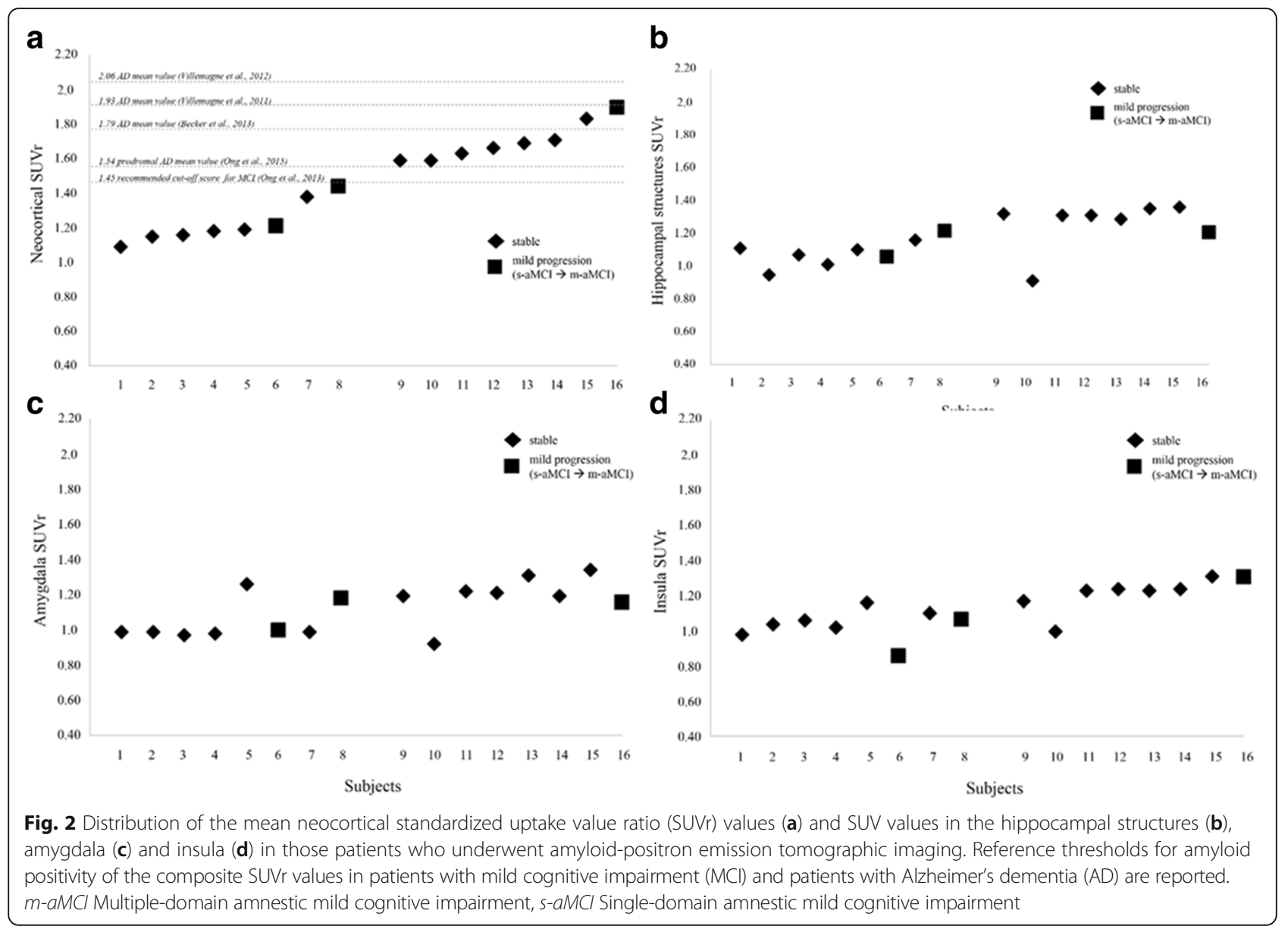

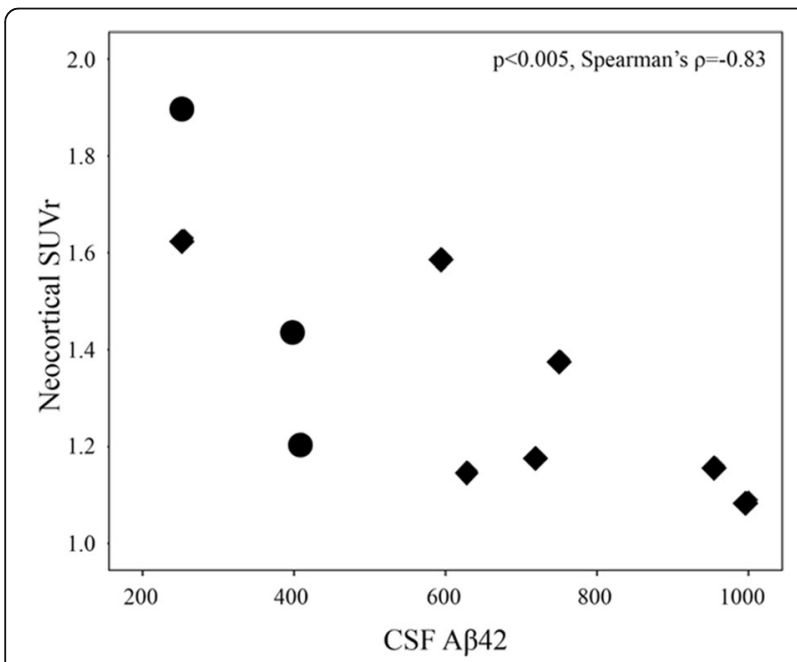

Fig. 3 Scatterplot showing the inverse correlation between mean neocortical standardized uptake value ratio (SUVr) and cerebrospinal fluid (CSF) $\beta$-amyloid (1-42) protein $\left(A \beta_{42}\right)$ values in those subjects who underwent both amyloid-positron emission tomography and lumbar puncture reduced CSF $A \beta_{42}$ values and no amyloid-PET burden, one had a normal CSF $A \beta_{42}$ value but a slight increase in SUVr value, and two had reduced CSF A $\beta_{42}$ levels and increased SUVr values. There was a significant negative correlation between mean neocortical SUVr and CSF $\mathrm{A} \beta_{42}$ values (Spearman's rho $=-0.83, p<0.005$ ).

\section{Discussion}

Our sample of subjects with aMCI with prevalent or exclusive long-term memory deficits and a slow cognitive progression did not show the FDG-PET hypometabolism pattern typical of AD. They had reduced glucose metabolism in the medial temporal lobe structures with no amyloid load visualised by PET imaging in these structures (see Figs. 1 and 2).

In addition to the consistent pattern of reduced metabolism in the hippocampal structures, hypometabolism in the frontomedial cortex, insula and anterior superior temporal cortex was present in some subjects. A few subjects showed reduction of glucose metabolism in the posterior cingulate cortex, which can be interpreted as a functional disconnection effect due to the severe involvement of the hippocampal structures. As reported in 
the literature, even in the absence of grey matter loss or amyloid toxicity in the posterior cingulate cortex, grey matter loss in the medial temporal lobe structures is sufficient to cause remote metabolic effects in connected regions [36].

This FDG-PET hypometabolic pattern involving limbic structures is similar to the pattern reported in some patients with bvFTD $[37,38]$. The subjects in our series, however, presented with a clinical phenotype of aMCI, and the additional mild behavioural changes found in many subjects did not fulfil the clinical criteria for bvFTD [39] at either onset or follow-up.

As for the amyloid load visualised by $\left[{ }^{18} \mathrm{~F}\right]$ florbetabenPET or CSF, we found evidence of $\beta$-amyloidosis in some subjects according to the amyloid-PET SUVr (i.e., 8 of 16 subjects showed intermediate or high SUVr values) and/ or CSF $A \beta_{42}$ cut-off values [19] (i.e., 10 of 20 showed low CSF $A \beta_{42}$ values). Notably, the cognitive profile and FDGPET features of subjects with evidence of in vivo $\beta$ amyloidosis did not show differences from patients with MCI without amyloid load (see Fig. 2 and Table 3). In addition, they did not progress to dementia during longterm follow-up (i.e., $58.3 \pm 10.1$ months). The clinical value of $\beta$-amyloidosis as a marker of prodromal $A D$ in these subjects is poor, however. It must be taken into consideration that a consistent percentage of cognitively normal subjects (i.e., $16-30 \%[27,32]$ ), as well as non-AD neurodegenerative patients [27], show incidental amyloid load with mean neocortical SUVr values above the proposed cut-off score for prodromal AD [40]. This is particularly true in elderly populations such as the one included in our study $[32,40]$. Thus, the $\beta$-amyloidosis seen in these subjects does not per se provide conclusive evidence for an "MCI due to AD" condition.

A pure or mixed limbic-predominant $\mathrm{AD}$ variant [12] could be the possible underlying neuropathological substrate in those subjects presenting with low cortical amyloid uptake. However, it is unlikely in patients with no evidence of brain amyloidosis. The possibility of other pathological substrates needs to be considered in the presence of long-lasting aMCI. These include AGD, a late-onset tauopathy typically characterised by tau lesions spreading throughout the limbic system $[5,6,41]$. AGD usually affects elderly subjects (mean age of onset 75-80 years), without sex differences, and is clinically characterised either by a severe amnestic syndrome with relative sparing of other cognitive functions $[5,6]$ or by a clinical neuropsychological phenotype overlapping with that of typical AD dementia.

Mixed neuropathological conditions have been suggested as a possible underlying substrate in patients with MCI with a stable clinical picture. Multiple co-morbid neuropathologies were reported previously in a large neuropathological study of individuals $(n=1337)$ followed longitudinally from normal or MCI status to death, showing that less than one-fourth of patients with MCI had "pure" $\mathrm{AD}$ at autopsy, whereas more than half of the stable patients with $\mathrm{MCI}$ had mixed AD pathology changes [42]. Brain arteriolosclerosis was also reported as a possible neuropathological substrate in this $\mathrm{MCI}$ case series [42].

No subject in our MCI sample progressed to dementia during the follow-up, notably not even the two patients who showed higher SUVr values. This finding also supports a non-AD condition overall in this subgroup and once more suggests a possible combined AGD and additional amyloid pathological substrate in those individuals with slightly elevated SUVr values. Some subjects with AGD indeed showed an associated AD-type pathology [6]. AGD and AD pathologies may act as additive factors, because subjects with combined AGD with mild to moderate $\mathrm{AD}$-type pathologies were more frequently associated with dementia progression than with pure AGD pathology [5, 43].

We found mild behavioural disturbances in the majority of our subjects with aMCI. Although the presence of emotional blunting could suggest a diagnosis of a bvFTD condition, no other behavioural changes typical of bvFTD (e.g., disinhibition; impulsiveness; perseverative, stereotypic or compulsive/ritualistic behaviour; hyperorality; and dietary changes) were present. Abnormal behaviours such as those observed in our sample represent common initial symptoms in pathology-confirmed AGD [44].

Although asymmetry of brain volume in medial temporal regions has been described in study of patients with advanced AGD $[45,46]$, this anatomostructural aspect was not reported by the expert radiologists who evaluated conventional MRI scans in our series. This work was indeed performed in a clinical setting, and advanced MRI measures were not available. Future studies are needed to highlight the role of subtle and specific anatomical changes in the differential diagnosis of this MCI group.

Other possible pathological substrates also need to be considered, such as primary age-related tauopathy (PART) [47] and TDP-43 pathology [48]. PART is a tauopathy in the absence of $\beta$-amyloidosis in which medial temporal lobe structures are primarily involved and abnormally elevated CSF tau protein levels are often observed [47]. Age-related deposition of TDP-43 protein is a neuropathological condition recently described in elderly people with impairments in episodic memory and no pathological diagnosis of $\mathrm{AD}$ or frontotemporal lobar degeneration [48]. According to Nag et al. [48], there is an association between this proteinopathy and age-related hippocampal sclerosis (i.e., reduction of the volume of CA1 and subiculum). Hippocampal sclerosis has been suggested by Bien et al. [49] as the main cause 
of memory loss in patients with MCI with predominant episodic memory impairment, slow progression of neuropsychological deficits and preservation of other cognitive functions. The large majority of elderly people with hippocampal sclerosis included in the Nag et al. cohort [48] had TDP-43 pathology.

Both PART and age-related TDP-43 proteinopathy can represent possible pathological substrates of the heterogeneous clinical spectrum called SNAP [9]. Reports on subjects with SNAP MCI [7-9, 14-17], as well subjects with PART [47] and age-related TDP-43 [48], indeed show some parallelism with the clinical features of our aMCI sample: advanced age, very mild cognitive symptoms, neurodegeneration without amyloidosis, and low risk of clinical neuropsychological decline. Only clinical and neuroimaging studies with neuropathological verification will provide conclusive results about the array of pathologies responsible for the clinical picture reported in such subjects with long-lasting stable amnestic MCI.

\section{Conclusion}

The main contribution of the present in vivo combined biomarker study (FDG-PET for neurodegeneration and amyloid-PET and CSF for pathology) is that additional nosographic classifications besides the limbic $\mathrm{AD}$ variant need to be considered in patients with $\mathrm{MCI}$ with a longlasting disease course and slowly progressing or nonprogressing cognitive decline.

\section{Acknowledgements}

We thank Dr. Michele Zamboni and Dr. Valeria Golzi for patient recruitment and Dr. Pierpaolo Alongi and Dr. Andrea Panzacchi for PET scan acquisition.

\section{Funding}

CC was funded by Fondazione Lilly (Eli Lilly grant 2011 "Imaging of neuroinflammation and neurodegeneration in prodromal and presymptomatic Alzheimer's disease phases"). This work was partially supported by European Union Seventh Framework Programme's Imaging of Neuroinflammation in Neurodegenerative Diseases (INMiND) Project FP7-HEALTH-2011 (grant agreement 278850) and by the CTN01_00177_165430 Project (IVASCOMAR COD CUP B88F13000420008). Piramal Imaging provided the NeuraCeq ${ }^{\mathrm{TM}}$ tracer used for amyloid-PET imaging.

\section{Availability of data and materials}

Owing to ethical restrictions protecting patient privacy, data are available upon request. Requests for access to an anonymised dataset may be sent to CC (cerami.chiara@hsr.it).

\section{Authors' contributions \\ CC and DP designed the study. AM, RS, SI and GM acquired clinical, neuropsychological and CSF data. LG acquired PET data. CC, AD and LP collected and analysed patient data. CC, AD and DP interpreted and drafted the manuscript. SFC supervised and revised the manuscript. All authors read and approved the final manuscript.}

\section{Ethics approval and consent to participate}

All patients gave written informed consent to the experimental procedures. Approval of the study design was obtained from the ethics committee of San Raffaele Hospital, Milan, Italy.

\section{Consent for publication}

Not applicable.

\section{Competing interests}

The authors declare that they have no competing interests.

\section{Publisher's Note}

Springer Nature remains neutral with regard to jurisdictional claims in published maps and institutional affiliations.

\section{Author details}

${ }^{1}$ Division of Neuroscience, San Raffaele Scientific Institute, Milan, Italy.

${ }^{2}$ Clinical Neuroscience Department, San Raffaele Turro Hospital, Milan, Italy.

${ }^{3}$ Neurology Department, San Raffaele Hospital, Milan, Italy. ${ }^{4}$ Nuclear Medicine Department, San Raffaele Hospital, Milan, Italy. ${ }^{5}$ NeTS Center, Istituto Universitario di Studi Superiori, Pavia, Italy. ${ }^{6}$ IRCCS S. Giovanni di Dio

Fatebenefratelli, Brescia, Italy. ${ }^{7}$ Università Vita-Salute San Raffaele, Milan, Italy.

Received: 24 July 2017 Accepted: 19 March 2018

Published online: 25 April 2018

References

1. Petersen RC. Mild cognitive impairment as a diagnostic entity. J Intern Med. 2004:256(3):183-94.

2. Sarazin M, Berr C, De Rotrou J, Fabrigoule C, Pasquier F, Legrain S, et al. Amnestic syndrome of the medial temporal type identifies prodromal AD: a longitudinal study. Neurology. 2007;69:1859-67.

3. Dubois B, Feldman HH, Jacova C, Hampel H, Molinuevo JL, Blennow K, et al. Advancing research diagnostic criteria for Alzheimer's disease: the IWG-2 criteria. Lancet Neurol. 2014;13(6):614-29.

4. Hornberger M, Piguet O, Graham AJ, Nestor PJ, Hodges JR. How preserved is episodic memory in behavioral variant frontotemporal dementia? Neurology. 2010;74(6):472-9.

5. Ferrer I, Santpere G, van Leeuwen FW. Argyrophilic grain disease. Brain. 2008;131(6):1416-32.

6. Tolnay M, Probst A. Argyrophilic grain disease. Handb Clin Neurol. 2008;89: 553-63.

7. Jack CR Jr, Knopman DS, Weigand SD, Wiste HJ, Vemuri P, Lowe V, et al. An operational approach to National Institute on Aging-Alzheimer's Association criteria for preclinical Alzheimer disease. Ann Neurol. 2012;71(6):765-75.

8. Wisse LE, Butala N, Das SR, Davatzikos C, Dickerson BC, Vaishnavi SN, et al. Suspected non-AD pathology in mild cognitive impairment. Neurobiol Aging. 2015;36(12):3152-62.

9. Jack CR Jr, Knopman DS, Chételat G, Dickson D, Fagan AM, Frisoni GB, et al. Suspected non-Alzheimer disease pathophysiology-concept and controversy. Nat Rev Neurol. 2016:12(2):117-24.

10. Marra C, Villa G, Quaranta D, Valenza A, Vita MG, Gainotti G. Probable Alzheimer's disease patients presenting as "focal temporal lobe dysfunction" show a slow rate of cognitive decline. J Int Neuropsychol Soc. 2012;18(1):144-50.

11. Cappa A, Calcagni ML, Villa G, Giordano A, Marra C, De Rossi G. Brain perfusion abnormalities in Alzheimer's disease: comparison between patients with focal temporal lobe dysfunction and patients with diffuse cognitive impairment. J Neurol Neurosurg Psychiatry. 2001;70(1):22-7.

12. Murray ME, Graff-Radford NR, Ross OA, Petersen RC, Duara R, Dickson DW. Neuropathologically defined subtypes of Alzheimer's disease with distinct clinical characteristics: a retrospective study. Lancet Neurol. 2011;10(9):785-96.

13. Whitwell JL, Dickson DW, Murray ME, Weigand SD, Tosakulwong N, Senjem ML, et al. Neuroimaging correlates of pathologically defined subtypes of Alzheimer's disease: a case-control study. Lancet Neurol. 2012;11(10):868-77.

14. Prestia A, Caroli A, van der Flier WM, Ossenkoppele R, Van Berckel B, Barkhof $\mathrm{F}$, et al. Prediction of dementia in $\mathrm{MCl}$ patients based on core diagnostic markers for Alzheimer disease. Neurology. 2013;80(11):1048-56.

15. Petersen RC, Aisen P, Boeve BF, Geda YE, Ivnik RJ, Knopman DS, et al. Mild cognitive impairment due to Alzheimer disease in the community. Ann Neurol. 2013:74(2):199-208.

16. Duara R, Loewenstein DA, Shen Q, Barker W, Potter E, Varon D, et al. Amyloid positron emission tomography with ${ }^{18} \mathrm{~F}$-flutemetamol and structural magnetic resonance imaging in the classification of mild cognitive impairment and Alzheimer's disease. Alzheimers Dement. 2013;9(3):295-301.

17. Caroli A, Prestia A, Galluzzi S, Ferrari C, van der Flier WM, Ossenkoppele R, et al. Mild cognitive impairment with suspected non-amyloid pathology (SNAP): prediction of progression. Neurology. 2015;84(5):508-15. 
18. Coan AC, Kubota B, Bergo FP, Campos BM, Cendes F. 3T MRI quantification of hippocampal volume and signal in mesial temporal lobe epilepsy improves detection of hippocampal sclerosis. AJNR Am J Neuroradiol. 2014; 35(1):77-83.

19. Sjogren $M$, Vanderstichele $H$, Agren $H$, Zachrisson O, Edsbagge $M$, Wikkelso C, et al. Tau and Aß42 in cerebrospinal fluid from healthy adults 21-93 years of age: establishment of reference values. Clin Chem. 2001;47(10): 1776-81.

20. Varrone A, Asenbaum S, Vander Borght T, Booij J, Nobili F, Någren K, et al. EANM procedure guidelines for PET brain imaging using $\left[{ }^{18} \mathrm{~F}\right] \mathrm{FDG}$, version 2. Eur J Nucl Med Mol Imaging. 2009;36(12):2103-10.

21. Della Rosa PA, Cerami C, Gallivanone F, Prestia A, Caroli A, Castiglioni I, et al. A standardized $\left[{ }^{18} \mathrm{~F}\right]$-FDG-PET template for spatial normalization in statistical parametric mapping of dementia. Neuroinformatics. 2014;12(4):575-93.

22. Perani D, Della Rosa PA, Cerami C, Gallivanone F, Fallanca F, Vanoli EG, et al. Validation of an optimized SPM procedure for FDG-PET in dementia diagnosis in a clinical setting. Neuroimage Clin. 2014;6:445-54.

23. Perani D, Cerami C, Caminiti SP, Santangelo R, Coppi E, Ferrari L, et al. Crossvalidation of biomarkers for the early differential diagnosis and prognosis of dementia in a clinical setting. Eur J Nucl Med Mol Imaging. 2016;43(3):499-508.

24. Cerami C, Della Rosa PA, Magnani G, Santangelo R, Marcone A, Cappa SF, et al. Brain metabolic maps in mild cognitive impairment predict heterogeneity of progression to dementia. Neuroimage Clin. 2014;7:187-94.

25. Presotto L, Ballarini T, Caminiti SP, Bettinardi V, Gianolli L, Perani D. Validation of ${ }^{18}$ F-FDG-PET single-subject optimized SPM procedure with different PET scanners. Neuroinformatics. 2017;15(2):151-63.

26. Bettinardi V, Presotto L, Rapisarda E, Picchio M, Gianolli L, Gilardi MC. Physical performance of the new hybrid PET / CT Discovery-690. Med Phys. 2011;38:5394-411.

27. Villemagne VL, Ong K, Mulligan RS, Holl G, Pejoska S, Jones G, et al. Amyloid imaging with ${ }^{18} \mathrm{~F}$-florbetaben in Alzheimer disease and other dementias. J Nucl Med. 2011:52(8):1210-7.

28. Lewczuk P, Zimmermann R, Wiltfang J, Kornhuber J. Neurochemical dementia diagnostics: a simple algorithm for interpretation of the CSF biomarkers. J Neural Transm. 2009;116(9):1163-7.

29. Lewczuk P, Kornhuber J, Network GDC, Toledo JB, Trojanowski JQ, KnapikCzajka $M$, et al. Validation of the Erlangen score algorithm for the prediction of the development of dementia due to Alzheimer's disease in predementia subjects. J Alzheimers Dis. 2015;48(2):433-41.

30. Shaw LM, Vanderstichele $H$, Knapik-Czajka M, Clark CM, Aisen PS, Petersen $\mathrm{RC}$, et al. Cerebrospinal fluid biomarker signature in Alzheimer's disease neuroimaging initiative subjects. Ann Neurol. 2009;65(4):403-13.

31. Rowe CC, Ackerman U, Browne W, Mulligan R, Pike KL, O'Keefe G, et al. Imaging of amyloid $\beta$ in Alzheimer's disease with ${ }^{18} \mathrm{~F}-\mathrm{BAY} 94-9172$, a novel PET tracer: proof of mechanism. Lancet Neurol. 2008;7(2):129-35.

32. Villemagne VL, Mulligan RS, Pejoska S, Ong K, Jones G, O'Keefe G, et al. Comparison of ${ }^{11} \mathrm{C}-\mathrm{PiB}$ and ${ }^{18} \mathrm{~F}$-florbetaben for $\mathrm{A} \beta$ imaging in ageing and Alzheimer's disease. Eur J Nucl Med Mol Imaging. 2012;39(6):983-9.

33. Ong KT, Villemagne VL, Bahar-Fuchs A, Lamb F, Chételat G. Raniga P, et al. ${ }^{18} \mathrm{~F}$-florbetaben $A \beta$ imaging in mild cognitive impairment. Alzheimers Res Ther. 2013;5(1):4

34. Ong KT, Villemagne VL, Bahar-Fuchs A, Lamb F, Langdon N, Catafau AM, et al $A \beta$ imaging with ${ }^{18} \mathrm{~F}$-florbetaben in prodromal Alzheimer's disease: a prospective outcome study. J Neurol Neurosurg Psychiatry. 2015;86(4):431-6.

35. Becker GA, Ichise M, Barthel H, Luthardt J, Patt M, Seese A, et al. PET quantification of ${ }_{18} \mathrm{~F}$-florbetaben binding to $\beta$-amyloid deposits in human brains. J Nucl Med. 2013;54(5):723-31.

36. Teipel S, Grothe MJ, Alzheimer's Disease Neuroimaging Initiative. Does posterior cingulate hypometabolism result from disconnection or local pathology across preclinical and clinical stages of Alzheimer's disease? Eur J Nucl Med Mol Imaging. 2016;43(3):526-36

37. Cerami C, Dodich A, lannaccone S, Marcone A, Lettieri G, Crespi C, et al. Right limbic FDG-PET hypometabolism correlates with emotion recognition and attribution in probable behavioral variant of frontotemporal dementia patients. PLoS One. 2015;10(10):e0141672.

38. Cerami C, Dodich A, Lettieri G, Marcone A, Lettieri G, Crespi C, et al. Different FDG-PET metabolic patterns at single-subject level in the behavioral variant of frontotemporal dementia. Cortex. 2016:83:101-12.

39. Rascovsky K, Hodges JR, Knopman D, Mendez MF, Kramer JH, Neuhaus J, et al. Sensitivity of revised diagnostic criteria for the behavioural variant of frontotemporal dementia. Brain. 2011;134(9):2456-77.
40. Chételat $\mathrm{G}$, La Joie R, Villain N, et al. Amyloid imaging in cognitively normal individuals, at-risk populations and preclinical Alzheimer's disease. Neuroimage Clin. 2013;2:356-65.

41. Braak H, Braak E. Cortical and subcortical argyrophilic grains characterize a disease associated with adult onset dementia. Neuropathol Appl Neurobiol. 1989;15:13-26.

42. Abner EL, Kryscio RJ, Schmitt FA, Fardo DW, Moga DC, Ighodaro ET, et al. Outcomes after diagnosis of mild cognitive impairment in a large autopsy series. Ann Neurol. 2017:81(4):549-59.

43. Thal DR, Schultz C, Botez G, Del Tredici K, Mrak RE, Griffin WS, et al. The impact of argyrophilic grain disease on the development of dementia and its relationship to concurrent Alzheimer's disease-related pathology. Neuropathol Appl Neurobiol. 2005;31(3):270-9.

44. Togo T, Isojima D, Akatsu H, Suzuki K, Uchikado H, Katsuse O, et al. Clinical features of argyrophilic grain disease: a retrospective survey of cases with neuropsychiatric symptoms. Am J Geriatr Psychiatry. 2005;13(12):1083-91.

45. Saito Y, Yamazaki M, Kanazawa I, Murayama S. Severe involvement of the ambient gyrus in a case of dementia with argyrophilic grain disease. J Neurol Sci. 2002;196(1-2):71-5.

46. Adachi T, Saito Y, Hatsuta H, Funabe S, Tokumaru AM, Ishii K, et al. Neuropathological asymmetry in argyrophilic grain disease. J Neuropathol Exp Neurol. 2010;69(7):737-44.

47. Crary JF, Trojanowski JQ, Schneider JA, Abisambra JF, Abner EL, Alafuzoff I, et al. Primary age-related tauopathy (PART): a common pathology associated with human aging. Acta Neuropathol. 2014;128(6):755-66.

48. Nag S, Yu L, Wilson RS, Chen EY, Bennett DA, Schneider JA. TDP-43 pathology and memory impairment in elders without pathologic diagnoses of AD or FTLD. Neurology. 2017:88(7):653-60.

49. Bien CG, Helmstaedter C, Elger CE. Is it really Alzheimer's disease? J Neurol Neurosurg Psychiatry. 2001;71(3):416-7.

\section{Submit your next manuscript to BioMed Central and we will help you at every step:}

- We accept pre-submission inquiries

- Our selector tool helps you to find the most relevant journal

- We provide round the clock customer support

- Convenient online submission

- Thorough peer review

- Inclusion in PubMed and all major indexing services

- Maximum visibility for your research

Submit your manuscript at www.biomedcentral.com/submit
Biomed Central 